\title{
Estrazione e raffinazione dello zolfo e paesaggio nella Sicilia romana tra IV e VI d.C.
}

\author{
Luca Zambito \\ Università di Palermo \\ email: lucazambito@libero.it
}

\begin{abstract}
Sulphur was important in several fields: military, tanning, wool's disinfection and many other activities. Nevertheless, the largest quantity of sulphur was used in agriculture, particularly in viticulture as it was used to combat fungal diseases especially in wine production areas. Sicily, and in particular the area surrounding Agrigento, was the main site of sulphur extraction. This built strong relationships with Rome and other agricultural centres in the Mediterranean Sea. Very important among the historical sources and the archaeological evidence are the so called Tabulae sulphuris, concerning the sulphur exploitation at the Agrigento's area. Recent finds and studies gave a new chronology dating to a few of the tegulae sulphuris and the related sulphur exploitation between the end of the fourth and the sixth centuries. It is now possible to attribute to Sicily (in particular, the "area solfifera") the trade relationships which arose in Classical times. We must connect these commercial and cultural ties to the export of wheat and other commodities. The main goal of this paper is to show new data on the exploitation structures and the transformation of the mining landscape between Late Antiquity and the Byzantine Age. During this time, Agrigentum lost its function as fiscal centre, maybe because other centres were acquiring this role.
\end{abstract}

Keywords: Sulfur Exploitation and Trade, Ancient Landscape, Survey, Roman Economy, Roman Sicily

\section{Introduzione}

In questo contributo ci si occuperà dell'aspetto del paesaggio minerario in età romana, dei markers della produzione del minerale e del rapporto fra le miniere e il porto di Agrigentum in età tardoantica. Si tratta di un lavoro cominciato da anni e che prevede un approccio multidisciplinare. Infatti, ad una prima fase in cui si sono indagati i campi di applicazione dello zolfo e quindi i mercati potenziali a cui poteva essere destinato, ha fatto seguito un'indagine sul campo per documentare le evidenze archeologiche delle attività estrattive e le strutture insediative ad esse correlate.

Fin da quando questa ricerca è stata concepita, si è riflettuto su alcuni tratti caratterizzanti le attività estrattive che si mostrano fortemente conservativi, tanto da rimanere costanti nel tempo. Si è appurato che tali attività non richiedevano un grande investimento tecnologico, avevano un notevole impatto sul territorio circostante e, di contro, altrettante ricadute positive; ancora, è stato accertato che era necessario un controllo diretto da parte dell'investitore al fine di evitare latrocini da parte delle figure intermedie nella catena estrattiva.

\section{Importanza del minerale sui mercati dell'antichità}

Grande fu il fabbisogno di zolfo sul mercato in età preindustriale, quando la richiesta di acido solforico per la produzione di soda generò una forte domanda e determinò la nascita, nel territorio 


\section{«Serie gessoso-solfifera»}

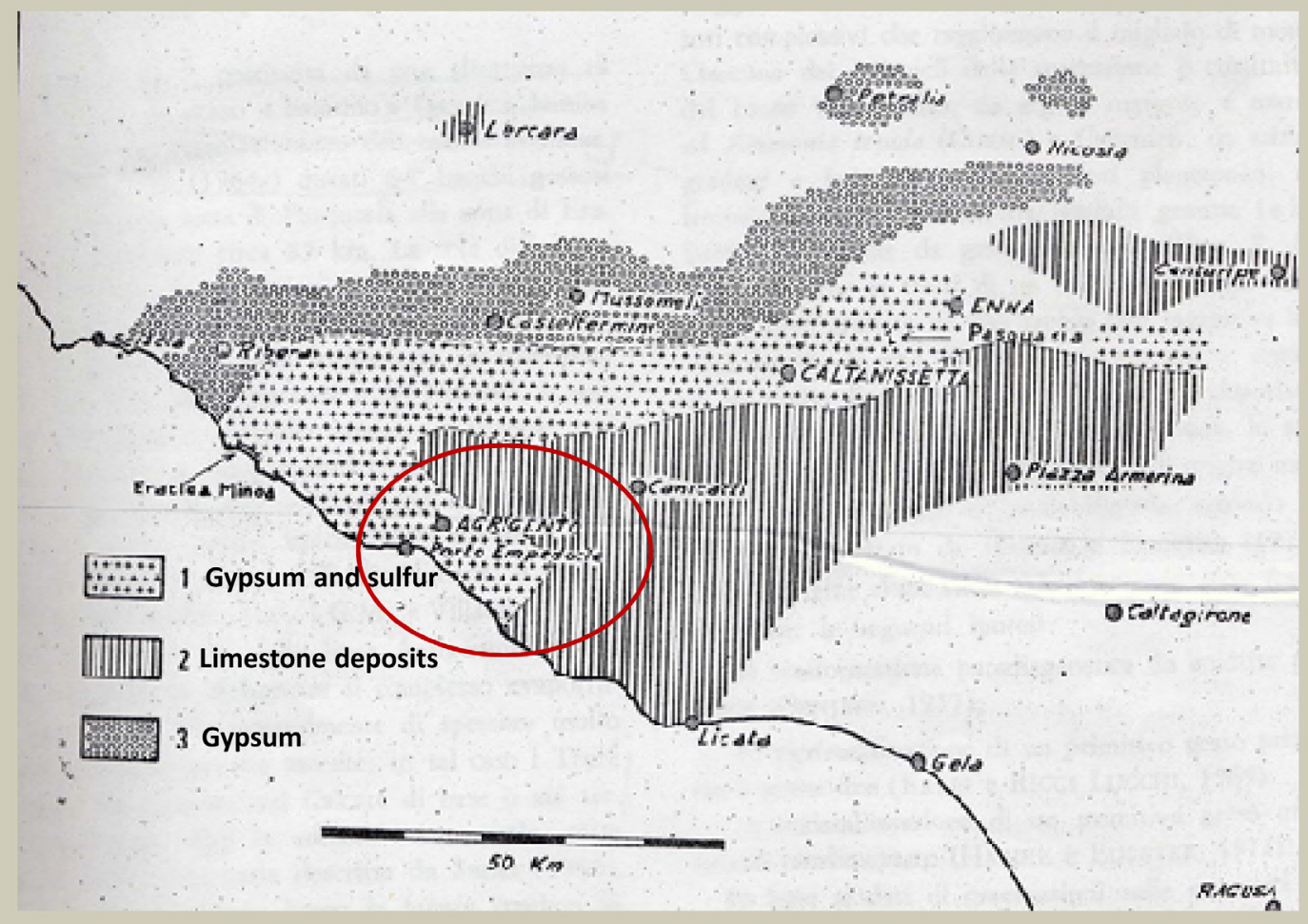

Fig. 1. Serie gessoso solfifera e l'area delle miniere romane (cerchiata in rosso).

agrigentino, di un gran numero di zolfare, che generarono un benessere economico diffuso e bloccarono, temporaneamente, i forti flussi migratori verso l'estero.

In età romana, le officine vetrarie, le fulloniche, le attività militari, quelle mediche e, infine, l'agricoltura e la scienza veterinaria richiedevano grandi quantità di zolfo (Zambito, 2010). Questa domanda fece in modo che Agrigentum fosse meta di navi onerarie destinate all'esportazione dello zolfo verso i mercati del Mediterraneo. Il minerale veniva esportato in grandi lingotti e non richiedeva contenitori peculiari come nel caso dell'allume eoliano e melio. Unico indicatore, quindi, della sua circolazione sono i pochi relitti che conservano traccia di lingotti di zolfo nel carico: quello elbano di Procchio, quello mazarese di Punta Granitola e due relitti sardi per cui abbiamo solo fugaci segnalazioni (Parker, 1992).

\section{Caratteristiche del paesaggio zolfifero}

Il territorio agrigentino è caratterizzato da grandi depositi di calcare zolfifero e gesso. La cosiddetta “Serie Gessoso solfifera”, nella quale si trova lo zolfo, copre una superficie di circa 1500 chilometri quadrati (fig. 1) ed è compresa fra le province di Agrigento, Caltanissetta ed Enna con un piccolo affioramento a Lercara Friddi (Palermo). Tuttavia, l'area interessata dai siti minerari romani sembra molto più ristretta e concentrata solo nel territorio a est di Agrigento.

Le particolari caratteristiche delle attività di estrazione e raffinazione determinarono e condizionarono le dinamiche insediative. Se, infatti, da un 


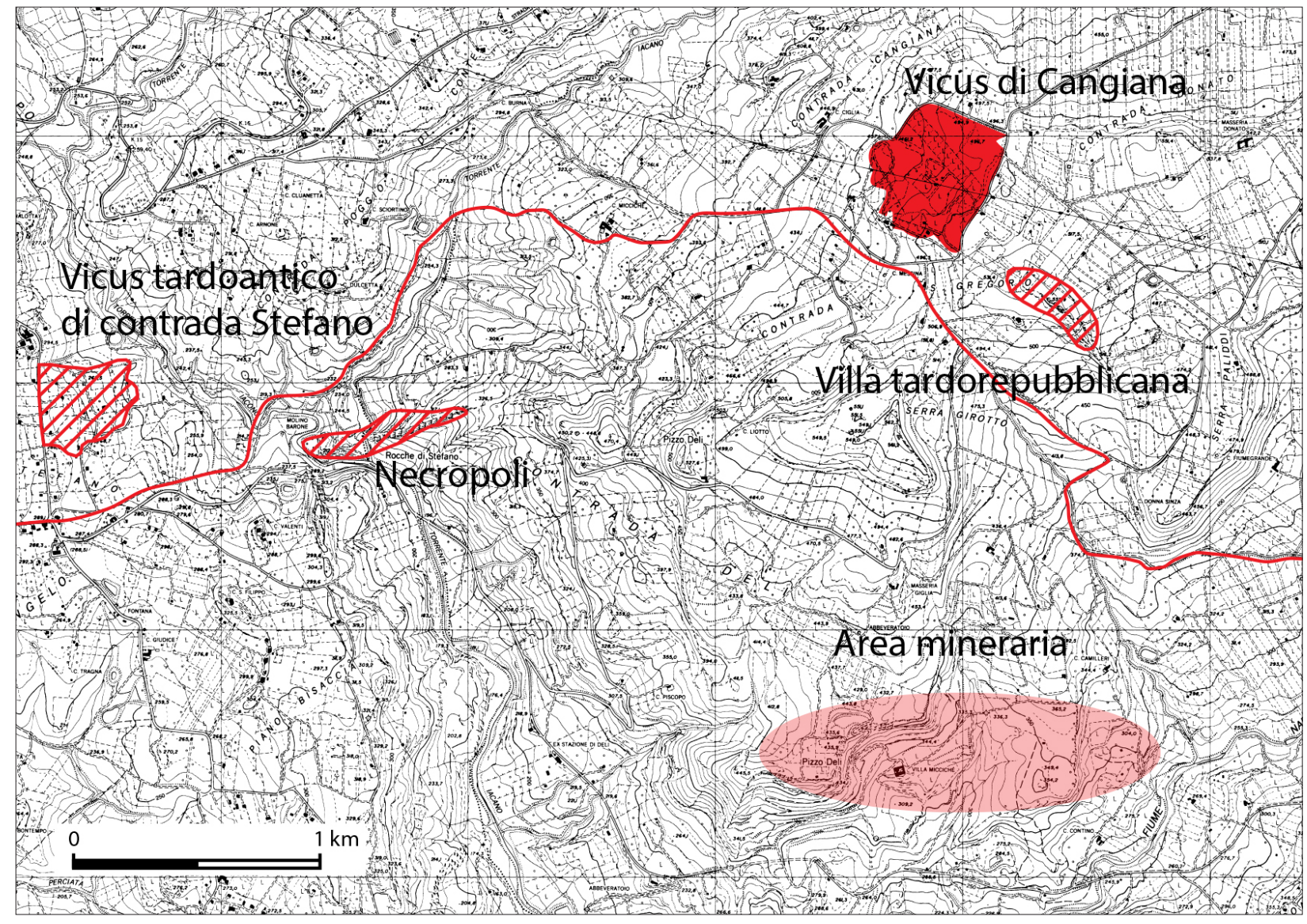

Fig. 2. Area attorno ai siti di Cangiana e Stefano e loro rapporto con il bacino minerario e la viabilità.

lato è necessario un diretto controllo delle attività lavorative e quindi una prossimità con le aree insediate, dall'altro lato, la produzione di anidride solforosa era dannosa per le coltivazioni, per il bestiame e direttamente per l'uomo. Le modalità insediative, quindi, dipendono dalla mediazione fra queste due esigenze. Se le attività di estrazione del minerale sono ad alto impatto, con tunnel e depositi all'aria aperta, il momento della raffinazione non prevede la costruzione di strutture di particolare complessità tecnologica: lo zolfo puro, infatti, si ottiene per autocombustione della ganga in grandi cumuli; il minerale, del resto, fonde a bassa temperatura e, per questo, non necessita di nessun apporto di altri combustibili.

Non era, perciò, necessario collocare gli stabil- imenti di raffinazione in prossimità di risorse boschive o idriche. A tal proposito, sono interessanti i documenti di archivio e le transazioni notarili ottocentesche da cui emerge, chiaramente, l'intento di tutelare le coltivazioni dagli effetti negativi della vicinanza alle zolfare ma che, altrettanto chiaramente, lasciano trasparire la precisa intenzione di privilegiare le seconde sugli interessi dei primi con permessi e deroghe rilasciate facilmente. Se, come abbiamo visto, l'impegno tecnologico richiesto in una zolfara era minimo, il suo impatto sulla natura del territorio e perfino sulla sua orografia era devastante: nuovi rilievi, costituiti da rosticcio, torrenti e corsi d'acqua deviati, condizionamento delle aree insediative e coltivate sono solo alcuni dei macrofenomeni, documentabili direttamente per l'età contemporanea, attraverso le fonti di archivio, per l'età basso-medievale e per la prima età moderna, e che vanno ricostruiti attraverso una attenta analisi dei dati, per l'antichità. 


\section{Descrizione del progetto di ricerca: metodologia-area di studio}

In sede di progettazione della ricognizione, finalizzata e da condurre su aree-campione, si è tenuto conto dei dati storici e topografici testimonianti l'esistenza di attività minerarie. Gli indizi sulla produzione mineraria antica, limitati alla presenza di tegulae sulphuris (fig. 2), portavano fin dall'inizio a concentrare le indagini nella porzione di territorio a est di Agrigento e fino a circa 40 chilometri da esso. Si è ragionato sulla distanza media percorribile, giornalmente, dagli addetti e, quindi, le aree-campione sono state delimitate tenendo conto dei seguenti due poli di attrazione: $i$ siti in cui erano state rinvenute le tegulae sulphuris da un lato e, dall'altro lato, i filoni zolfiferi in cui cercare l'imbocco delle miniere.

Nove le aree campione iniziali: Casalvecchio, Fico, Quattro Finaite, Firrìo (nel territorio comunale di Racalmuto), Cignana (nel territorio di Palma di Montechiaro), Punta Bianca, Stefano, S. Gregorio (nel territorio di Favara), Puzzu Rosi (nel territorio comunale di Comitini) i cui confini sono stati disegnati tenendo conto di precisi confini topografici (l'esistenza di particolari caratteristiche orografiche e altimetriche, la presenza di bacini fluviali, l'esistenza di grandi arterie viariel e analizzando il loro rapporto con i filoni solfiferi. Si sono documentate anche le discenderie, i forni e le strutture relative alle zolfare moderne rinvenute nelle aree della ricognizione così come si sono registrate anche le attività secondarie, sia contemporanee che successive rispetto alle zolfare: estrazione e lavorazione del gesso, produzione di beni e strumenti funzionali alla gestione delle miniere, cantieri di recupero del minerale esausto e cave di calcare. Tutte queste attività hanno ovviamente pesato e condizionato, quando non reso impossibile, l'analisi dei dati sulle miniere romane.

Si è deciso fin dall'inizio di effettuare ricognizioni ripetute e in stagioni diverse approfittando, in alcuni casi, di condizioni meteo-climatiche particolari (Belvedere, 1994: 75 con bibliografia precedente). Solitamente non si è superato il numero di due ricognitori; le ottime condizioni di visibilità hanno consentito di coprire giornalmente una vasta area (nell'ordine dei quattro ettari per giorno/personal con una distanza reciproca fra i ricognitori di 8-10 m (Plog, Plog \& Wait, 1978: 38994). Nelle aree interessate da sterpaglie e in quelle in cui i rilievi collinari si facevano particolarmente importanti, si è camminato a distanze ridotte segnalando eventuali tracce di anfratti, tombe e, soprattutto, di manufatti legati ad attività produttive moderne.

I forni per la raffinazione dello zolfo hanno conosciuto, nei secoli, poche variazioni: dalle semplici calcarelle, accumuli di zolfo all'aria aperta, si passò ai più complessi calcheroni, cumuli di minerale coperti da rosticcio esausto. Infine, e solo a partire dalla fine dell' 800 , si diffuse il cosiddetto forno Gill che permetteva di riutilizzare il calore prodotto dalla fusione per altri forni, consentendo, così, un risparmio di minerale.

Particolarmente utile si è rivelata, infine, una lunga indagine di archivio che in alcuni casi (come quello di Racalmutol ha consentito di restituire il contesto di rinvenimento per le tegulae. Gli esemplari racalmutesi furono rinvenuti, infatti, alle porte del centro moderno e la loro scoperta era stata annotata da un erudito locale nel 1897. Un altro caso è quello relativo alla tegola da Grotte, oggi conservata presso il Museo di Palermo, riguardo alla quale si conservava solo la generica indicazione "trovata a mezzo miglio a tramontana dalla stazione di Grotte'. In questo caso la ricerca sul terreno ha permesso, con il rinvenimento di un frammento riconducibile allo stesso tipo, di rintracciare con esattezza l'area di Firrìo che, appunto, si trova a circa $800 \mathrm{~m}$ a nord dalla stazione di Grotte.

\section{Primi dati dalla ricerca}

Se le fonti letterarie e la documentazione archeologia sono sostanzialmente "mute" riguardo all'estrazione dello zolfo siciliana, non c'è dubbio che ci troviamo di fronte a uno "speaking landscape". L'indagine topografica è servita, infatti, a 
dare una definizione di spazio e paesaggio minerario. Ci si è chiesti quali sono le peculiarità delle attività estrattive e cosa sappiamo delle modalità di sfruttamento minerario nell'antichità. In realtà abbiamo pochi dati che trasmettano indicazioni sul lavoro nelle fodinae: in questo caso un testimone imprescindibile, anche, per la sua conoscenza diretta dell'argomento, è Plinio il Vecchio (Lana, 1990: 453-502). Gli addetti all'estrazione vengono solitamente poco considerati da Plinio e sono indicati solo di rado con la loro qualifica: operati, fossores, vigiles, operae.

Ad ogni modo, dal passo pliniano, si evincono alcuni importanti dati sull'organizzazione del lavoro nei filoni minerari spagnoli: i turni erano di dieci ore e gli addetti, nelle ore di riposo, non uscivano alla luce del sole ma organizzavano sotto terra la pausa: 'cuniculis per magna spatia actis cavantur montes lucernarum ad lumina; eadem mensura vigiliarum est, multisque mensibus non cernitur dies'. Il dato è analogo a quello che si ricava dalla Lex Metallis di Vipasca in cui è documentato anche l'uso, una volta esaurita una vena, di procedere all'allargamento del fronte di cava: partendo proprio dai vecchi putei si procedeva all'escavazione di cunicoli laterali (Plin., NH, 33, 70).

Un secondo modello di gestione delle miniere è conservato in Diodoro Siculo $(5,35-38)$, il quale documenta la gestione delle miniere egiziane e i massacranti turni continui a cui erano sottoposti gli schiavi:

gli schiavi impegnati nel lavoro in miniera producono ai loro padroni profitti di incredibile entità; essi sotto terra nelle gallerie, logorando il loro fisico giorno e notte, in molti muoiono per l'eccessivo patimento; non hanno diritto a riposo né a pausa del lavoro mal costretti dalle frustate dei sorveglianti a sopportare la terribilità dei loro mali, gettano via infelicemente la vita e la sofferenza è prolungata per quelli che resistono più a lungo a causa del loro vigore fisico e della loro forza d'animo. Per loro la morte è preferibile alla vita, tanto grande è la sofferenza (Lana, 1990: 48087).

Il colorito malsano dei minatori, un tratto topico, è dovuto tanto alla loro esposizione a polveri, vapori e altri agenti inquinanti (sia naturali quanto prodotti dalla stessa escavazionel quanto alla lunga permanenza sottoterra. In epoca moderna gli zolfatai erano pendolari: dai centri urbani si dirigevano alle bocche di miniera e, finito il turno, vi facevano ritorno. Nei casi in cui le miniere erano particolarmente lontane si aveva un pendolarismo settimanale, tuttavia le dannosissime attività di raffinazione dello zolfo, che producendo anidride solforosa rendevano il territorio inadatto a qualsiasi altra attività e, quasi alla vita stessa, erano strettamente legate agli imbocchi delle miniere. Analogamente il proprietario della licenza o l'affittuario doveva controllare con frequenza l'attività in cui, spesso, aveva investito tutte le sue liquidità. $\mathrm{Ci}$ si chiede, quindi, se questo genere di insediamento potesse avere, in qualche maniera, un antecedente di epoca romana.

Uno degli obiettivi iniziali del survey è stato verificare sul campo se tale asserzione avesse un fondamento. Il paesaggio minerario è "liquido" per definizione, è infatti possibile trovarvi cave di rosticcio esausto, usato per il fondo stradale o come inerte nelle fondazioni delle abitazioni; sono molto comuni, inoltre, grandi cave di calcare e ciò ha ovviamente un grande impatto sulle caratteristiche orografiche della zona. Ancora, di solito, nei depositi geologici di zolfo si trovano tracce dell'estrazione del gesso: tagli nella roccia, blocchi pronti per essere estratti e numerose fornaci per la cottura della pietra (calcare). Frequenti, infine, anche altre strutture più semplici, come i forni da pane, per il sostentamento degli operai.

All'interno di ciascuna area si è effettuato, pertanto, un survey intensivo, documentando il record ceramico e, in taluni casi, le emergenze architettoniche. Sono stati percorsi complessivamente circa undici chilometri quadrati e sono state individuate 35 UT: tra queste, tre sono siti produttivi (Piano di Corsa, Lucia e Cozzo Tondo), in cui la raffinazione del minerale è testimoniata da uno strato di zolfo accumulato sulle tegulae sulphuris rinvenute e da evidenti segni di contatto col fuoco e con alte temperature. Altri lCignana, PianoVento, Punta Bianca, Stefano, Saraceno, Firrìo, Quattro Finaite) sembrano interpretabili come siti residen- 


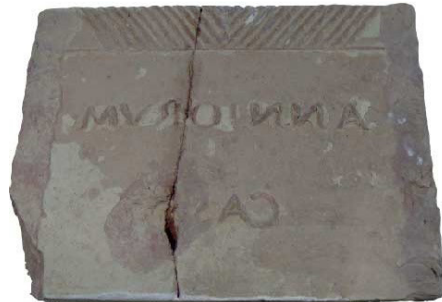

1

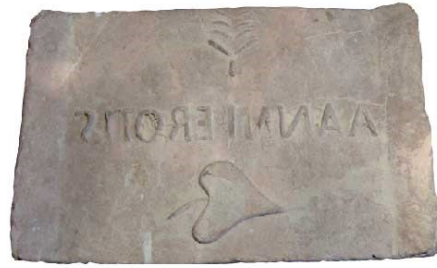

4

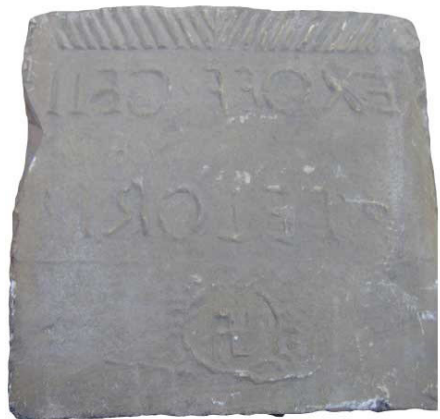

6

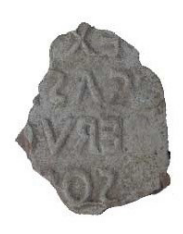

2

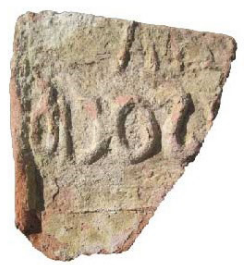

3

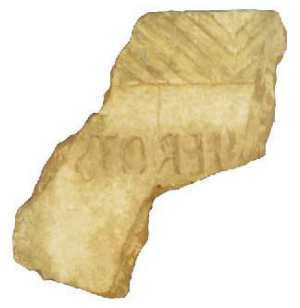

5

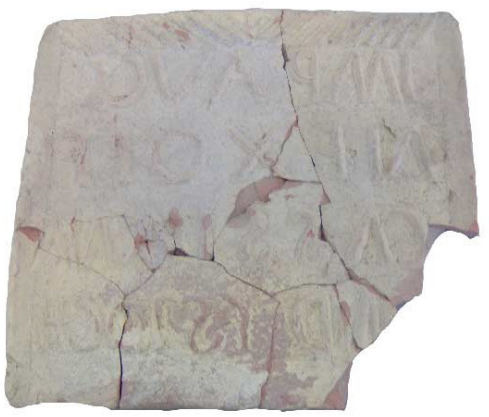

7
Fig. 3. Tegulae di Gellius Pelorus.

ziali; nei restanti casi si tratta di necropoli.

Prima di questa ricerca non si era in grado di inserire le tegulae sulphuris in una griglia crono-tipologica lesse erano indistintamente assegnate ad un periodo compreso fra il II e il IV secolo d.C.I. In alcuni casi, invece, si è potuto assegnare ciascun gruppo di tegulae sulphuris al singolo sito o contesto topografico e, quindi, ridefinire meglio la datazione della tegola sulla base del record ceramico attestato.

\section{Conclusioni e prospettive di ricerca}

L'analisi del record ceramico e della sua distribuzione consente di delineare, per la produzione dello zolfo, un range cronologico molto ampio che va dalla tarda età ellenistica ad epoca bizantina. Gli abitati di epoca imperiale sembrano qualificarsi tutti come residenze di pregio, probabilmente legate alla figura del dominus dell'impresa mineraria. I siti interpretabili come villae cessano di essere frequentati entro la prima metà del III secolo d.C. mentre, a partire dalla fine del IV d.C., si osserva, nell'area della nostra ricerca, la diffusione di una 


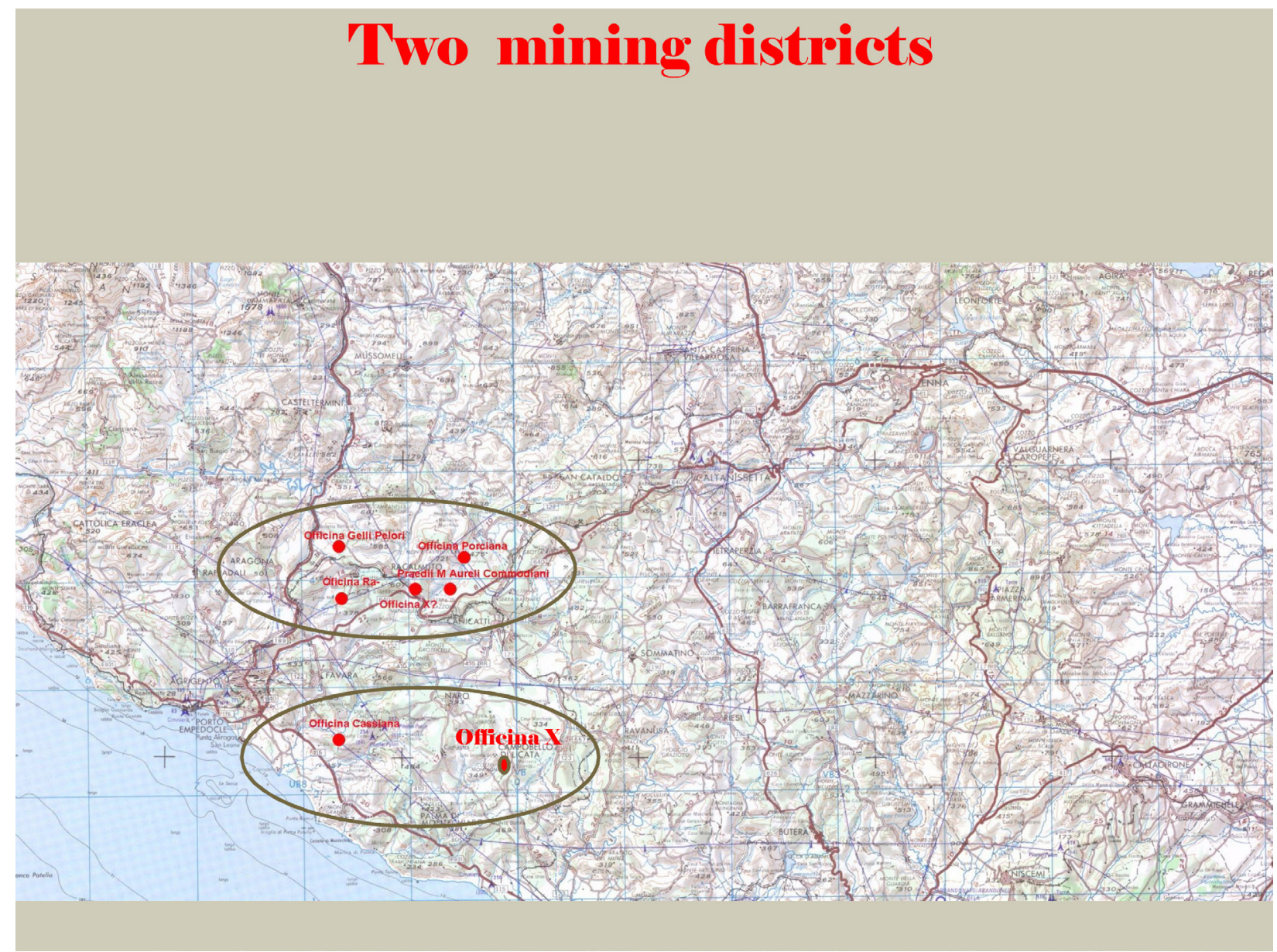

Fig. 4. Distribuzione delle officinae, localizzate in due distretti principali

nuova forma insediativa: il villaggio. All'interno di questi nuovi insediamenti, inoltre, sono presenti indicatori di molteplici attività artigianali e produttive: produzione di calce, vetro, ferro e grandi impianti per la produzione di vino e olio.

È possibile, ora, ricostruire alcuni settori del paesaggio minerario e disegnare una carta delle infrastrutture, primarie e secondarie, funzionali all'estrazione, alla raffinazione e all'esportazione del minerale (tunnel, aree abitate, assi viari, aree di necropoli). Certo, rimane ancora elevato il numero di tegulae sulphuris, conservate presso il Museo di Agrigento, per cui non conosciamo ancora il contesto produttivo. Continuare l'indagine topografica in aree solfifere diverse, potrebbe sicuramente ampliare le nostre consocenze su quei distretti. Ma è senza alcun dubbio all'indagine stratigrafica che devono essere affidate le prospettive di una migliore comprensione dell'articolazione interna delle officinae e della loro evoluzione nel tempo.

Nella ricostruzione proposta, tuttavia, c'è una grande assenza: si tratta del personale che, direttamente lavorava all'estrazione. Dalle fonti sappiamo che doveva essere di condizione schiavile oppure si trattava di condannati per gravi reati. Stupisce, comunque, la completa assenza di qualsiasi traccia di frequentazione nei tre siti individuati come sedi di zolfare (Zambito, 2014b) e di impianti di raffinazione del minerale. Esisteva un pendolarismo giornaliero verso l'abitato? Oppure, piuttosto, gli operai trascorrevano tutto il loro tempo sul luogo di lavoro e, per questo, in superficie non se ne rinviene traccia?

La ricerca ha riportato alla luce gli elemen- 
ti principali del paesaggio minerario, da epoca romana fino ad età bizantina. In pochi fortunati casi è stato possibile anche connettere la singola tegula sulphuris alla singola produzione e, in ultima analisi, ad uno e un solo distretto produttivo con la sua area di raffinazione. Dei circa 35 tipi differenti di tegulae sulphuris almeno 20 rimangono ancora da assegnare a un preciso contesto topografico. Questo dato indica quanto e verso quale direzione la ricerca potrebbe continuare.

Alcuni fortunati rinvenimenti hanno consentito di stabilire dove si trovasse l'officina Cassiana: tra Miniera Lucia e Ciavolotta, due importanti zolfare moderne all'interno del bacino del fiume Naro. Anzi, fu, con ogni probabilità, proprio la presenza di canyons generati dal fiume a rendere più semplice l'individuazione dei filoni da sfruttare. Un secondo centro minerario si sviluppò attorno a Racalmuto (Ag). A nord del vallone di Racalmuto, a fine ' 800 , furono rinvenuti alcuni esemplari di tegulae sulphuris con tracce di contatto con zolfo e che documentano la presenza della officina Porciana. A sud-ovest del centro moderno, invece, è stata ubicata l'officina $R a$ - lancora oggi riesce difficile integrare il toponimol. Ancora, a nord-est, tra Racalmuto e Milena, sono da individuare le proprietà di Marcus Aurelius Commodianus. Sulle colline a nord di Grotte è da collocare la presenza della officina Gelli Pelori (fig. 3).

L'area mineraria antica pare, dunque, concentrarsi in due principali distretti: il primo, a est-nordest di Agrigento, oggi fra i comuni di Racalmuto, Grotte e Milena; il secondo, costiero, nel bacino del fiume Naro, pochi chilometri a sud-est di Agrigento (fig. 4).

Agrigentum sembra aver perso, nel corso del VI d.C., la sua funzione di centro fiscale di riferimento a favore di altri centri economici dell'hinterland che si affermarono durante la tarda antichità e la prima età bizantina; tuttavia l'estrazione dello zolfo non sembra aver conosciuto una soluzione di continuità.

Un centro anonimo, in prossimità di Racalmuto, acquisì tale ruolo come pare documentato da numerosi dati convergenti, quali la scoperta di un importante tesoretto di aurei, databile a metà del

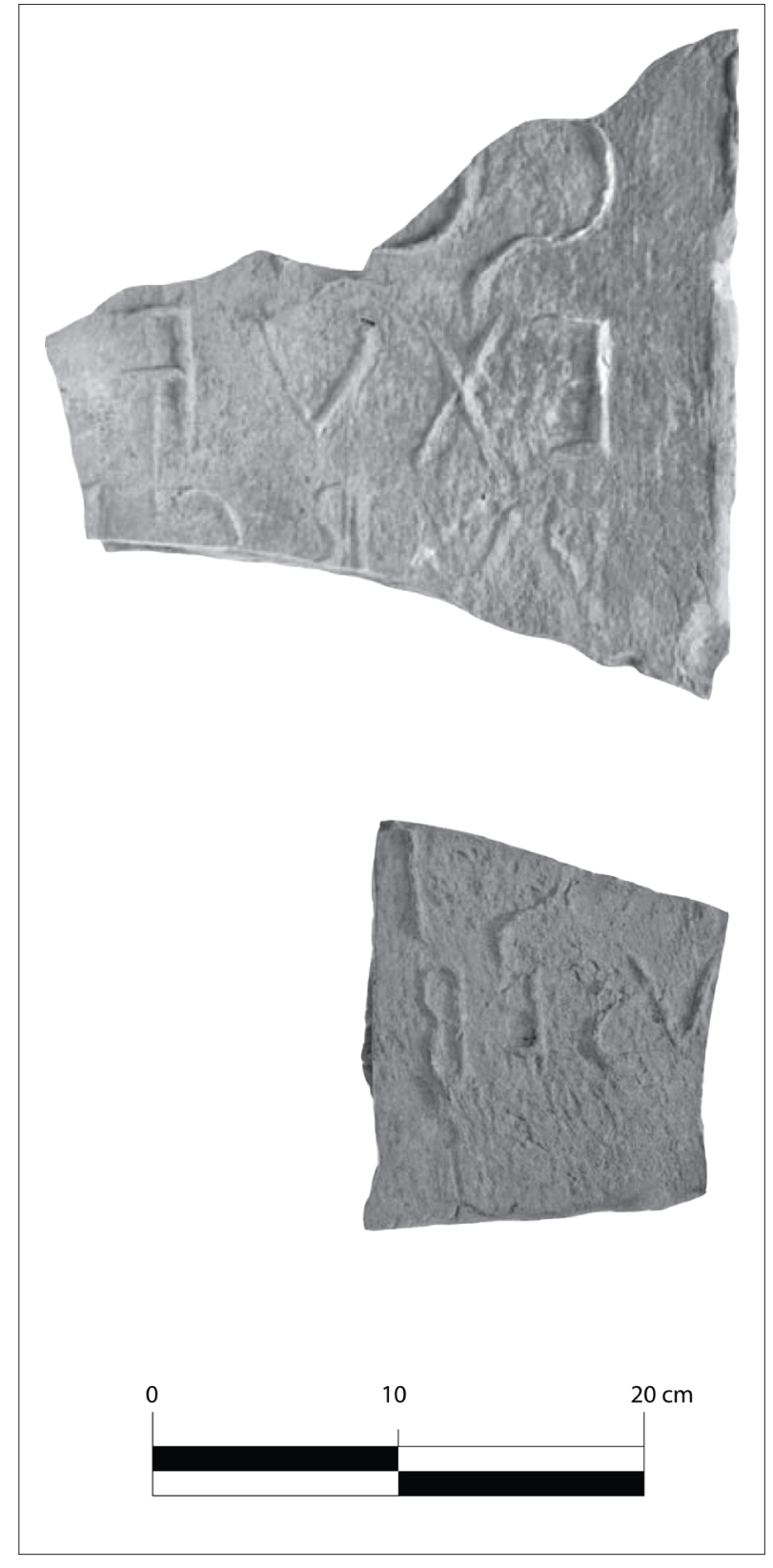

Fig. 5. Tegula bizantina di Eusebius.

VII d.C., un sigillo di metà VIII d.C., che testimonia la presenza di interessi di un certo Antiochos Notarios funzionario bizantino e, non ultima, la tegola di Eusebius (fig. 5), che si propone di datare ad età bizantina, sulla base dell'iscrizione e del fatto che non sia stata prodotta ad Agrigento, come tutte le altre, ma nei dintorni di Racalmuto stessa (Zambito, 2014a). 
Infine, recenti indagini archeologiche nel porto di Agrigento sembrano confermare la grande vitalità da esso mantenuta almeno dal VII d.C. e, senza soluzione di continuità, fino agli inizi del IX d.C. Il commercio dello zolfo potrebbe essere la chiave per spiegare la valenza strategica di questo approdo e aver costituito un cespite economico su cui Agrigentum contò per un lungo periodo.

\section{Bibliografia}

Arcifa L \& F Tomasello 2007: La via dello zolfo: insediamento e viabilità nel territorio di Milena (CL), in Micciché C, Modeo S \& Santagati L (ed.), La Sicilia romana tra repubblica e alto impero, Atti del Convegno di Studi, Caltanissetta 20-21 maggio 2006: 199-209. Sciacia, Caltanissetta.

Belvedere 0, 1994: La ricognizione sul terreno, Journal of Ancient Topography, 4, 69-84.

Healy JF, 1993: Miniere e metallurgia nel mondo greco e romano, (ed.) L Pirzio Biroli Stefanelli, trad. MJ Strazzulla, L'Erma di Bretschneider, Roma, volume originale pubblicato 1978.

Lana I, 1990: La condizione dei minatori nelle miniere secondo Plinio il Vecchio e altri autori antichi, in Lana I (ed.), Sapere lavoro e potere in Roma antica: 453-502. Jovene, Napoli.

Plog S, F Plog \& W Wait 1978: Decision making in Modern Surveys, in Schiffer MB (ed), Advances in Archaeological Method and Theory, vol. 1: 383-421. Academic Press, New York.

Parker AJ, 1992: Ancient Shiprecks of the Mediterranean and the Roman Provinces, Tempus Reparatum, Oxford.

Salmeri G, 1992: Miniere di zolfo in Sicilia ed in Grecia in età imperiale, in Salmeri G, Sicilia romana. Storia e storiografia: 29-43. G. Maimone, Catania.

Wilson RJA, 1990: Sicily under the Roman Empire: the archaeology of a Roman province, 36 b.C.-a.D. 535, Aris \& Phillips, Warminster.

Zambito L, 2010: Lo sfruttamento dello zolfo in età romana. Gentes Senatoriae e attività mineraria in Sicilia, PhD thesis, Università di Messina.

Zambito L, 2014a: Nuovi dati sulle tegulae sulphuris. A proposito di due nuovi esemplari da Racalmuto
(Ag), Zeitschrift für Papyrologie und Epigraphik, 188, 261-64.

Zambito L, 2014b: Produzione e commercio dello zolfo ad Agrigentum e nel suo territorio in Caminneci V (ed.), Le opere e i giorni: Lavoro, produzione e commercio tra passato e presente: 225-44. Sarcuto, Agrigento. 
